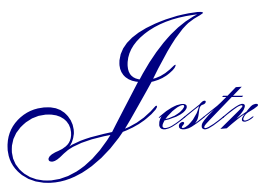

Lecture Note

www.jestr.org

\title{
What is a surface excess?
}

\author{
A. Ch. Mitropoulos* \\ Department of Petroleum Technology, Cavala Institute of Technology, St. Lucas 65404 Cavala.
}

Received 19 October 2007; Accepted 14 January 2008

\begin{abstract}
J. Willard Gibbs in his pioneering work on the influence of surfaces discontinuity upon the equilibrium of heterogeneous masses suggested for the measurement of the quantities of a system a geometrical surface dividing the interfacial layer. Surface excess is the difference between the amount of a component actually present in the system, and that which would be present in a reference system if the bulk concentration in the adjoining phases were maintained up to the arbitrary chosen but precisely determined in position dividing surface.
\end{abstract}

Keywords: surface excess, Gibbs adsorption.

Adsorption of a component at the phase boundary of a system results to a different concentration in the interfacial layer than that in the adjoining bulk phases. The adsorbate density and composition profiles within that layer cannot be measured by today's technology; the actual amounts adsorbed are not meaningful experimental variables. To resolve this problem Gibbs [1] suggested that: " ...it will be convenient to be able to refer to a geometrical surface, which shall be sensibly coincident with the physical surface of discontinuity, but shall have a precisely determined position. For this end, let us take some point in or very near to the physical surface of discontinuity, and imagine a geometrical surface to pass through this point and all other points which are similar situated with respect to the condition of the adjacent matter. Let this geometrical surface be called the dividing surface."

Surface excess [2] is the difference between the amount of a component actually present in the system, and that which would be present in a reference system if the bulk concentration in the adjoining phases were maintained up to a chosen geometrical dividing surface [3]; i.e. as though the interface had no effect. Schematically:

$$
\left[n_{i}\right]_{\text {surface }}=\left[n_{i}\right]_{\text {real }}-\left[n_{i}\right]_{\text {reference }} .
$$

where $\left[\mathrm{n}_{\mathrm{i}}\right]_{\text {surface }}=\mathrm{n}_{\mathrm{i}}{ }^{\sigma}$ is the surface excess amount [4] of component (i); $\left[n_{i}\right]_{\text {real }}=n_{i}$ is the total amount of that component in the real system; and $\left[n_{i}\right]_{\text {reference }}=n_{i}{ }^{a}+n_{i}{ }^{\beta}$ is the amount of the same component (i) in a reference system having volume $\mathrm{V}$ equal to the real system which is divided by a hypothetical surface into

\footnotetext{
* E-mail address: amitrop@teikav.edu.gr

ISSN: 1791-2377 @ 2008 Kavala Institute of Technology. All rights reserved.
}

volumes $V^{\alpha}+V^{\beta}=V$ each one assumed to contain $C_{i}^{\alpha} V^{\alpha}=n_{i}^{\alpha}$ and $\mathrm{C}_{\mathrm{i}}^{\beta} \mathrm{V}^{\beta}=\mathrm{n}_{\mathrm{i}}^{\beta}$ moles with $\mathrm{C}_{\mathrm{i}}^{\alpha}$ and $\mathrm{C}_{\mathrm{i}}^{\beta}$ the bulk concentrations in the real system [5], respectively. More formally:

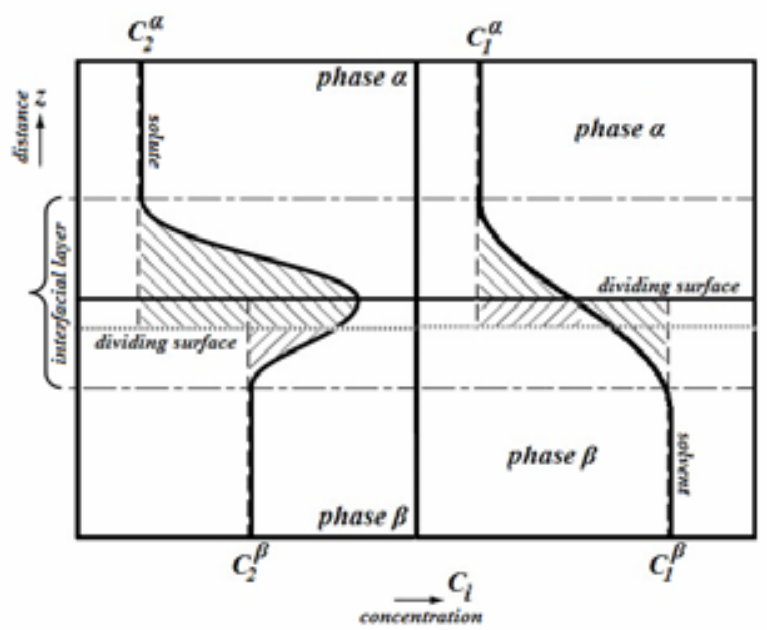

Figure 1. Concentration profiles of a binary system as a function of distance normal to the phase boundary. Bold curved lines, in both frames, are the concentration profiles of the solute and the solvent in the real system, respectively, and again vertical broken lines are the concentrations in the reference system (being actually the extend of the bulk concentrations up to the dividing surface). Chain dotted lines indicate the boundaries of the interfacial layer. Bold horizontal line is the dividing surface and dotted horizontal line is another choice for the location of the dividing surface. The surface excess is the sum of the shaded areas above and under the dividing surface. Notice that in the case of the solvent by choosing the upper dividing surface (a suitable one) the resulted surface excess is zero, whereas by choosing the lower one surface excess is not zero. 


$$
n_{i}^{\sigma}=n_{i}-C_{i}^{a} V^{a}-C_{i}^{\beta} V^{\beta}
$$

If $A_{s}$ is the area of the interface [6], the areal surface excess concentration $\Gamma_{\mathrm{i}}^{\sigma}$ is defined as:

$$
\Gamma_{i}^{\sigma}=\frac{n_{i}^{\sigma}}{A_{s}}
$$

In a binary system, where (1) is e.g. the solvent and (2) is the solute and by (') is denoted a second choice for the dividing surface (see Fig.1), can be easily shown that:

$$
\Gamma_{2}^{\sigma}-\Gamma_{1}^{\sigma}\left[\frac{C_{2}^{a}-C_{2}^{\beta}}{C_{1}^{a}-C_{1}^{\beta}}\right]=\Gamma_{2}^{\prime \sigma}-\Gamma_{1}^{\prime \sigma}\left[\frac{C_{2}^{a}-C_{2}^{\beta}}{C_{1}^{a}-C_{1}^{\beta}}\right]
$$

Since the location of the dividing surface was arbitrary chosen, the above equation can only be true if each side separately equals a constant [7]; that is, the relative adsorption of component (2) with respect to component (1): $\Gamma_{2}{ }^{1}$. Obviously, a symmetric definition of $\Gamma_{1}^{2}$ exists too [8]. One way to determine experimentally $\Gamma_{2}{ }^{1}$ is to locate the dividing surface at a position where $\Gamma_{1}{ }^{\sigma}=0$; i.e. the reference system contains the same amount of component (1) as the real one. In this case eq.(4) reduces to $\Gamma_{2}{ }^{1}=\Gamma_{2}{ }^{\sigma}$. If however is not clear where to locate the dividing surface, $\Gamma_{2}{ }^{1}$ may still be calculated by rearranging eq.(4) such as:

$$
\Gamma_{2}^{1}=\frac{n_{2}-C_{2}^{a} V}{A_{s}}-\frac{n_{1}-C_{1}^{a} V}{A_{s}} \times \frac{C_{2}^{a}-C_{2}^{\beta}}{C_{1}^{a}-C_{1}^{\beta}} .
$$

The quantities on the right hand side of eq.(5) are all directly measurable, provided that $\mathrm{A}_{\mathrm{s}}$ is known.
A fundamental equation for surfaces of discontinuity between fluid masses at constant temperature is given by Gibbs having, in the case of a binary system, the form:

$$
d \sigma=-\Gamma_{1}^{\sigma} d \mu_{\imath}-\Gamma_{2}^{\sigma} d \mu_{2}
$$

where $\sigma$ is the surface tension, and $\mu_{1}$ and $\mu_{2}$ are the chemical potentials of the adjacent phases. By deciding to choose a dividing surface such as $\Gamma_{1}{ }^{\sigma}=0$, eq.(6) takes the form:

$$
d \sigma=-\Gamma_{2}^{1} d \mu_{2}
$$

According to this equation [9] for a system involving a solvent and a solute, there is an excess surface concentration of solute if the solute decreases the surface tension and a deficient surface concentration of solute if the solute increases the surface tension.

Direct measurements of surface excess quantities were carried out by several investigators. McBain [10] constructed a microtome device consisted of a sharp blade, Salley et al. [11] developed a tracer method where the solute is labeled with a radioisotope, and Smith [12] studied the ellipticity of light reflection from the thickness of an adsorbed film on mercury. Application of Gibbs adsorption isotherm on electrolyte solution was introduced by Wagner [13].

In this note an elementary review on the concept of surface excess was given. It must pointed out, however, that even in the frame of formal surface thermodynamics the characteristic quantities involved need further specification in order to became operational and again need different specification for each different type of interface (e.g. sold/gas [14], etc).

Acknowledgments: The author would like to thank Archimedes research Project and INTERREG III "Hybrid Technology for Separation" for funding this work

\section{References and Notes}

1. J.W.Gibbs, The Collected Works of J. W. Gibbs, Longmans, Green, New York, 1931, Vol. I, p. 219. See also: E.A.Guggenheim and N.K.Adam, Proc.Roy.Soc. (London) A139, 218 (1933); E.A.Guggenheim, Trans.Faraday Soc. 36, 397 (1940); R.C.Tolman, J.Chem.Phys. 16, 758 (1948); R.C.Tolman, J.Chem.Phys. 17, 118 (1949); E.H.Lucassen-Reynders, Prog.Surf.Membrane Sci. 10, 253 (1976); G.Schay, A comprehensive presentation of the thermodynamics of adsorption excess quantities, Pure Appl. Chem. 48, 393 (1976); A.W.Adamson, Physical Chemistry of Surfaces, Wiley, New York (1982).

2. D.H.Everett, Definitions Terminology and Symbols in Colloid and Surface Chemistry, Pure Appl. Chem. 31, 577 (1972); D.H.Everett, Pure Appl. Chem. 53, 2181 (1981); D.H.Everett, in Colloidal Dispersion, J.W.Goodwin (ed), The Royal Society of Chemistry, London (1982); D.H.Everett, Reporting Data on Adsorption from Solution at the Solid/Liquid Interface, Document prepared for the IUPAC Commission meeting in Copenhagen (1985); D.H.Everett, Reporting data on adsorption from solution at the solid/solution interface, Pure Appl. Chem. 58, 967 (1986); D.H.Everett, Application of thermodynamics to interfacial phenomena, Pure Appl. Chem. 59, 45 (1987).

3. Also referred as Gibbs dividing surface (GDS) or Gibbs surface; IUPAC 31, 588 (1972).

4. Surface excess is an algebraic quantity and may be positive (excess) or negative (deficiency). Also referred as Gibbs adsorption: IUPAC: 31, 588 (1972). A former term for the surface excess was superficial density (see Gibbs in Ref.1).
5. For liquid/vapour interfaces, at low vapor pressures, $\mathrm{Ci} \beta$, where $\beta$ is the gaseous phase, may be neglected.

6. Interface is the plane ideally marking the boundary between two phases. Interphase, however, is a different term which refers to the interfacial layer; being the inhomogeneous space region intermediate between two bulk phases in contact, and where properties are significantly different from, but related to, the properties of the bulk phases. When the interfacial layer is regarded as a phase it is called interphase; IUPAC: 58, 439 (1986).

7. Since $\Gamma_{2}{ }^{1}$ is independent form the location of the dividing surface it is possible to dispense the geometric interpretation of excess quantities and formulate a suitable algebraic method; i.e. without explicit reference to a dividing surface. For both geometric and algebraic methods see: Ref.2; R.S.Hansen, J.Phys.Chem. 66, 410 (1962); F.C.Goodrich, Trans. Faraday Soc. 64, 3403 (1968); F.C.Goodrich, in Surface and Colloid Science, E.Matijevic and F.R.Eirich (eds), Wiley, New York (1969); R.Delay, I.Prigogine, A.Bellemans, D.H.Everett, Surface Tension and Adsorption. Longmans, London (1966); R.J.Good, Thermodynamics of adsorption and Gibbsian distance parameters in two and three-phase systems, Pure Appl. Chem. 48, 427 (1976); R.J.Good, Thermodynamics of Adsorption and Gibbsian Distance Parameters: The Pressure Coefficient of Interfacial Tension in Ternary Two- and Three-Phase Systems, J. Colloid Interface Sci. 85, 128 (1982); R.J.Good, Thermodynamics of Adsorption and Gibbsian Distance Parameters: Interfacial Distances and the Surface Excess Volume, J. Colloid Interface Sci. 85, 141 (1982); R.J.Good, J. Colloid Interface 
Sci. 110, 298 (1986); K.J.Motomura, Comments on: Thermodynamics of Adsorption and Gibbsian Distance Parameters by R.J.Good, J. Colloid Interface Sci. 110, 294 (1986); K.J.Motomura, Thermodynamic Studies on Adsorption at Interfaces: General Formulation, J. Colloid Interface Sci. 64, 348 (1978); Z. Kiraly and I. Dekany, Algebraic and geometric interpretations of adsorption excess quantities, Colloid Polym. Sol. 266, 663 (1988); Z. Kiraly and I. Dekany, Interpretation of adsorption excess quantities: the absolute surface excess concentration, Colloid Polym. Sci. 268, 687 (1990).

8. Other excess amount similar to the relative adsorption is the molar reduced adsorption $\Gamma_{2}{ }^{\mathrm{n}}$ and the volumetric reduced adsorption $\Gamma_{2}{ }^{\mathrm{V}}$. For more details see: D.H.Everett, IUPAC manual of symbols and terminology for physicochemical quantities and units, Washington (1971).

9. Referred also as Gibbs adsorption isotherm.

10. J.W.McBain and C.W.Humphreys, J.Phys.Chem. 36, 300 (1932); J.W.McBain and R.C.Swain, Proc.Roy.Soc. (London) A154, 608 (1936).

11. D.J.Salley, A.J.Weith Jr, A.A.Argyle, and J.K.Dixon, Proc.Roy.Soc. (London) A203, 42 (1950); J.K.Dixon, C.M.Judson, and D.J.Salley,
Monomolecular Layers, American Association for the Advancement of Science, Washington (1954). See also: K.Tajima, M.Muramatsu, and T.Sasaki, Bull.Chem.Soc. Japan 43, 1991 (1970); K.Tajima, Bull.Chem.Soc.Jap. 43, 3063 (1970); K.Sekine, T.Seimiya, and T.Sasaki, K.Tajima, M.Muramatsu, and T.Sasaki, Bull.Chem.Soc.Japan 43, 629 (1970); K.Tajima, M.Iwahashi, and T.Sasaki, Bull.Chem.Soc.Jap. 44, 3251 (1971); N.H.Steiger and G.Aniansson, J.Phys.Chem. 58, 228 (1954); K.Shinoda and K.Ito, J.Phys.Chem. 65, 1499 (1961); S.J.Rehfeld, J.Colloid Interface Sci. 31, 46 (1969).

12. T.Smith, J.Colloid Interface Sci. 28, 531 (1968).

13. C.Wagner, Phys. Z. 25, 474 (1924). See also: L.Onsager and N.N.T.Samaras, J. Chem. Phys. 2, 528 (1934); L.Yan, J.Stat.Phys. 110,825 (2003).

14. See e.g. S.Sircar, Measurement of Gibbsian Surface Excess, AIChE J. 47, 1169 (2001); S.Sircar, Gibbsian Surface Excess for Gas Adsorptions, Ind.Eng.Chem.Res. 38, 3670 (1999); A.L.Myers, Thermodynamics of Adsorption in Porous Materials, AIChE J. 48, 145 (2002). 